\title{
Tumor Vessel Up-Regulation of INSR Revealed by Single-Cell Expression Analysis of the Tyrosine Kinome and Phosphatome in Human Cancers
}

\author{
Sara Kiflemariam, Viktor Ljungström, Fredrik Pontén, and Tobias Sjöblom
}

From the Science for Life Laboratory, Department of Immunology, Genetics and Pathology, Uppsala University, Uppsala, Sweden

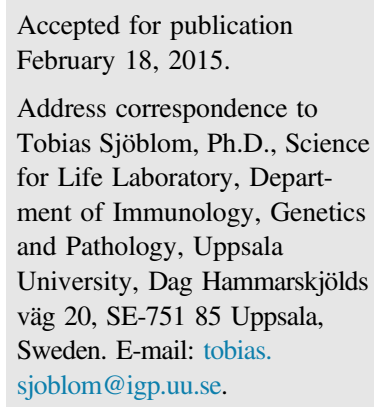

\begin{abstract}
The tyrosine kinome and phosphatome harbor oncogenes and tumor suppressor genes and important regulators of angiogenesis and tumor stroma formation. To provide a better understanding of their potential roles in cancer, we analyzed the expression of 85 tyrosine kinases and 42 tyrosine phosphatases by in situ hybridization 48 human normal and 24 tumor tissue specimens. Nine-tenths of the assessed transcripts had tumor cell expression concordant with expression array databases. Further, pan-cancer expression of AATK, PTPRK, and PTPRU and expression of PTPRS in a subset of tumors were observed. To demonstrate tumor subcompartment resolution, we validated the predicted tumor stroma-specific markers HTRA1, HTRA3, MXRA5, MXRA8, and SERPING1 in situ. In addition to known vascular and stromal markers such as PDGFRB, we observed stromal expression of PTK6 and TNS1 and vascular expression of INSR, PTPRF, PTPRG, PTPRU, and TNS1, of which INSR emerged as a tumor-specific vessel marker. This study demonstrates the feasibility of large-scale analyses to chart the transcriptome in situ in human cancers and their ability to identify novel cancer biomarkers. (Am J Pathol 2015, 185: 1600-1609; http://dx.doi.org/10.1016/j.ajpath.2015.02.019)
\end{abstract}

Certain gene families, such as the tyrosine kinases and tyrosine phosphatases, are known to be frequent targets of mutations in human cancers. ${ }^{1-3}$ Tyrosine phosphorylation, governed by the balanced action of tyrosine kinases and phosphatases, is essential to many cellular processes that regulate transcription, cell cycle progression, proliferation, differentiation, and apoptosis. ${ }^{4,5}$ The tyrosine kinome harbors several proto-oncogenes, such as $A B L, E R B B 2$, $F G F R 1, K I T, N T R K 3$, and PDGFRB, and is the focus of intense drug discovery efforts. ${ }^{6-11}$ Similarly, somatic lossof-function mutations in tyrosine phosphatases were identified in several common human cancers, for example, PTPN3, PTPN13, PTPN14, PTPRF, PTPRG, and PTPRT in colorectal cancers. ${ }^{2}$ Kinases and phosphatases such as vascular endothelial growth factor receptors, PDGFRB, fibroblast growth factors, TIE, BMX, and PTPRB also play important roles in angiogenesis, an essential process in tumor development. ${ }^{12-17}$ This explains the intense interest in drug discovery to target kinases and phosphatases and the need to systematically map their expression in human normal and malignant tissues.
Advances in sequencing technologies have enhanced our ability to find putative cancer genes, leading to a surge in the number of genes mutated in $1 \%$ to $5 \%$ of patient tumors. ${ }^{18-20}$ To unambiguously identify these genes as bona fide cancer genes they must be linked to tumor phenotypes by functional studies. However, few universal experimental approaches enable characterization of large gene sets, leading to a substantial investment in each gene studied. One essential aspect is the gene expression pattern, which can help prioritize genes that are expressed in the malignant cells for further studies. Expression array studies and transcriptome analyses have generated extensive knowledge on the gene expression levels of the human transcriptome in normal and diseased tissues. Several different resources such as MediSapiens offer aggregated and normalized data from large sets of gene expression arrays that can provide a reference for whole

Supported by the Beijer Foundation (T.S.), the Swedish Foundation for Cancer Research grant CAN 2009/1017 (T.S.), and the Swedish Foundation for Strategic Research grant FFL-3, F06-0050 (T.S.).

Disclosures: None declared. 
organ or whole tumor gene expression profiles. ${ }^{21}$ Further, initiatives such as the Allen Human Brain Atlas have increased the understanding of human brain function and the impact of genetic disorders on brain structures. ${ }^{22}$ These efforts provide excellent references to validate in situ expression analyses of human genes.

Although systematic charting of the transcriptome in situ was performed in the developing mouse, no such effort was performed in the context of human tissues despite the inherent scalability and specificity of in situ hybridization (ISH). ${ }^{23}$ To enable such efforts, we have adapted automated mRNA ISH for expression analysis of large gene sets in human formalin-fixed, paraffin-embedded (FFPE) tissue arrays. ${ }^{24}$ To better understand which cell types of normal tissues and tumors express different tyrosine kinases and phosphatases, we analyzed their in situ expression pattern in 48 different normal human tissues and tumors of the colorectum, breast, prostate, kidney, bladder, and testis. In combination with existing large-scale expression data sets, this provides a comprehensive and validated expression map of the human tyrosine kinome and phosphatome and enables the discovery of novel compartment-specific cancer biomarkers.

\section{Materials and Methods}

\section{Reference Data Sets}

To obtain reference gene expression data with cell type resolution during organism development and to obtain expression data from human organs and tumors, we i) collated annotated GenePaint database (http://www.genepaint.org; last accessed February 8, 2015) ISH data (release July 5, 2012), ii) mined the literature for ISH gene expression studies, and iii) summarized phenotypes and expression patterns from published genetargeting studies in the mouse. Further, we used the MediSapiens database (http://ist.medisapiens.com, last accessed February 8, 2015) that encompasses 157 normalized gene expression array studies with 9783 human tissue specimens from 43 normal tissue types and 68 tumor types to obtain bulk tissue gene expression data from the adult human ${ }^{21}$ (Supplemental Tables S1-S3). We were unable to find annotated, publicly available developmental gene expression data at cell type resolution for one-third of tyrosine kinases and phosphatases.

\section{Large-Scale ISH on FFPE Human Tissue}

Forty-eight arrayed normal adult human tissues in triplicate and 24 cancers each of breast, colorectum, prostate, kidney, bladder, and testis were used for each gene. All tissues were obtained from Uppsala University Hospital (Uppsala, Sweden), placed in neutral buffered ( $\mathrm{pH} 7.4$ ) 4\% formaldehyde after the surgical procedure. The time between ligation of blood flow and fixation was estimated to be 15 minutes to 1 hour, and the fixation time was 1 to 4 days. After fixation, the tissue was cut into pieces, dehydrated, infiltrated with paraffin, and assembled on tissue microarray blocks. These blocks were cut in $4-\mu \mathrm{m}$ sections and used for ISH and immunohistochemistry (IHC). ${ }^{25}$

ISH on tissue arrays was performed as described. ${ }^{24}$ Essentially, FFPE tissue arrays were deparaffinized in xylene and permeabilized by proteinase K treatment. PCR primers for generation of approximately 500-nt products were designed for each gene. The PCR products were used to generate digoxigenin-labeled antisense RNA probes by in vitro transcription. The tissue sections were incubated with the RNA probes (Supplemental Table S4) for 4 hours. After hybridization, high-stringency washes and several blocking steps were performed to remove unbound probe and to block endogenous enzyme activities. The bound RNA probes were detected by chromogenic antidigoxigenin IHC. Whole slide images were digitized at 40-fold magnification by using an Aperio ScanScope CS Slide Scanner System (Leica Biosystems, Buffalo Grove, IL). For each tissue, gene expression was annotated to tumor cell compartment, vascular, connective tissue stroma, and inflammatory cells as either strong, intermediate, or absent. Previously, we evaluated the use of sense RNA probes as negative controls and independent antisense RNA probes for validation of gene expression patterns. ${ }^{24} \mathrm{We}$ concluded that the best approach here would be to use publicly available resources and databases such as PubMed, GenePaint, and MediSapiens and also independent RNA probes for validating the gene expression of the tyrosine kinases and phosphatases.

\section{Comparisons between MediSapiens and ISH}

By comparison with MediSapiens data, we estimated that a MediSapiens signal intensity of 200 arbitrary units is likely to produce a signal also in the ISH analysis. ISH expression patterns were considered concordant with MediSapiens expression patterns if the tumor type with the highest ranked expression level in MediSapiens also showed positivity in one or more of such tumors in ISH, the rank order was conserved between MediSapiens and ISH such that tumor types that had higher levels in MediSapiens had ISH expression, and when tumor types with lower expression rank lacked ISH expression. Genes with median MediSapiens expression level $\leq 200$ in all but three or fewer examined tissues were considered not expressed/expressed at low levels. We defined genes with outlier expression levels in MediSapiens as having a group of observations with at least twice the median expression level and an expression level $\geq 1000$ in one or more forms of cancer.

\section{In Silico Prediction of Novel Tumor Stroma-Specific Genes}

Serial analysis of gene expression (SAGE) libraries for transcripts enriched in breast cancer-associated myofibroblasts compared with normal myofibroblasts were obtained from 
Allinen et $\mathrm{al}^{26}$ through the Cancer Genome Anatomy Project. ${ }^{27}$ The Digital Gene Expression Displayer tool (http://cgap.nci. nih.gov/tissues/gxs, last accessed October 31, 2014) was used to identify SAGE tags with at least fourfold enrichment in the cancer myofibroblast library. Duplicate gene entries and genes with tag count $<20$ were removed, and the remaining genes were annotated with UniProt (http://www.uniprot.org, last accessed February 8, 2015). Entries with subcellular location secreted or transmembrane and no previous information on involvement in cancer in the literature were investigated further. Secreted or transmembrane proteins with potential growth factor activity, enzymatic activity, or receptor activity were selected for ISH validation.

\section{IHC}

Tissue sections were deparaffinized, hydrated in graded ethanol, and blocked for endogenous peroxidase activity. Heat-induced antigen retrieval was performed by boiling the glass slides in citrate buffer, $\mathrm{pH} 6.0$, for 4 minutes at $125^{\circ} \mathrm{C}$. Automated IHC was performed as described previously. ${ }^{28}$ Briefly, tissue sections were blocked and rinsed in wash buffer. Primary affinity-purified polyclonal antibody was incubated on the slides, followed by incubation with a secondary anti-rabbit antibody conjugated to a horseradish peroxidase-labeled polymer. The signal was developed with diaminobenzidine mixed with chromogen. The tissues were counterstained with Mayers hematoxylin.

\section{Results}

\section{Gene Expression Reference to the Kinome and Phosphatome}

To obtain a comprehensive reference on tissue expression, we compiled gene expression data from prior ISH efforts in the mouse, literature on knockout characterization and developmental studies in the mouse, and normalized transcriptome analyses of human tissues and tumors for 85 genes of the tyrosine kinome and 42 genes of the tyrosine phosphatome (Supplemental Tables S1-S3). The tyrosine kinome has been more extensively studied than the phosphatome, which is revealed by the numbers of publications per gene (median, 487; range, 8 to 29,077 for the kinases versus median, 61; range, 1 to 9207 for the phosphatases). For these genes, we obtained normalized human array expression data $(96 \%$ versus $95 \%$ of genes), annotated GenePaint mouse developmental expression patterns (22\% versus 17\%), developmental gene expression literature (56\% versus 55\%), and published mouse knockouts (96\% versus 64\%) (Supplemental Tables S1-S3).

\section{In Situ Analysis of Gene Expression in Arrayed Human Normal and Tumor Tissues}

We next generated ISH probes to all genes of the tyrosine kinome and phosphatome (median length, 515 nucleotides;

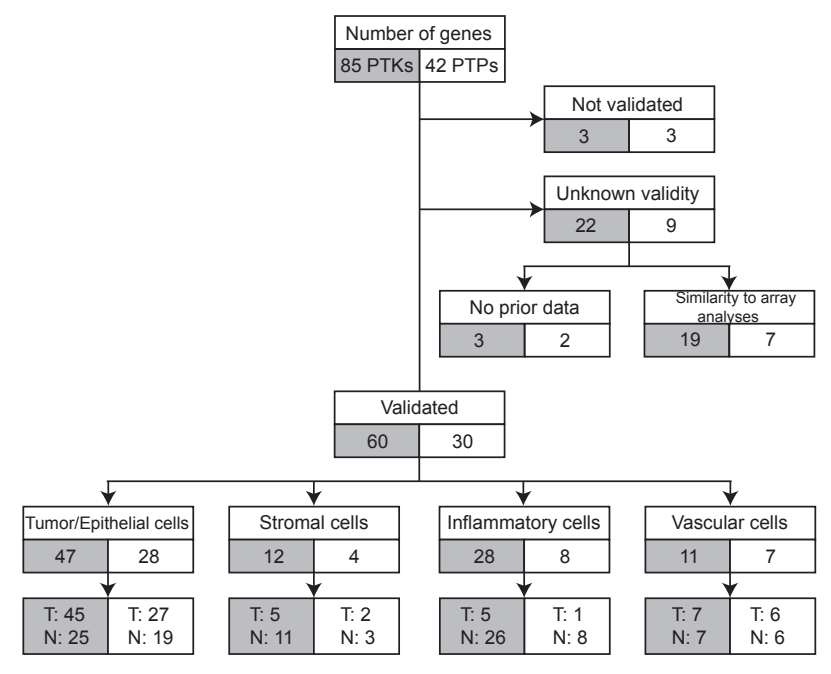

Figure 1 Summary of compartment-specific gene expression analysis of 127 genes of the tyrosine kinome and phosphatome. The in situ gene expression pattern of 85 PTKs and 42 PTPs were evaluated in human normal tissues and cancers of the breast, colorectum, prostate, bladder, kidney, and testis. Six genes had expression patterns discordant with reference gene expression data. Further, 31 genes could not be validated because no reference data were available or their observed expression levels and reference database expression levels were low. Ninety genes had clear expression signals in accordance with validation data sources. of these, 75 displayed epithelial or tumor cell expression, 18 vascular expression, 16 stromal expression, and 36 inflammatory cell expression, in normal or tumor tissues. N, normal; PTK, protein tyrosine kinase; PTP, protein tyrosine phosphatase; $\mathrm{T}$, tumor.

range, 255 to 595 nucleotides) (Supplemental Table S4). Valid RNA probes could not be generated for the tyrosine kinases FGFR3, LTK, and SRMS and for the tyrosine phosphatases MPPED1, PTPN5, PTPN7, and PTPRR because of multiple splice variants and/or short sequences, which led to their exclusion from analysis. Chromogenic ISH of arrayed FFPE tissue specimens was performed, comprising 48 arrayed normal adult human tissues in triplicate and 24 cancers each of the breast, colorectum, prostate, kidney, urinary bladder, and testis for each of the 127 genes. The stained tissue specimens were scored for expression in the epithelial or tumor cell compartments and in the vascular, stromal, and inflammatory cell compartments (Supplemental Tables S5 and S6). By comparison with published literature and databases, we sought to determine the validity of gene expression patterns (Figure 1). One-fifth of genes showed expected low or undetectable expression in normal tissues and the tumor tissues studied here (tyrosine kinases $A B L 2, A L K, B M X$, DDR2, EPHA3, EPHA5, EPHA6, EPHA10, FER, FRK, INSRR, JAK2, LMTK2, MUSK, NTRK1, NTRK2, ROS1, $T E C$, and TXK and tyrosine phosphatases $M T M 1, M T M R 3$, PTPDC1, PTPN2, PTPN14, PTPN21, PTPN22, PTPN23, and $P T P R H$ ), which was confirmed by largely negative ISH analyses. In addition, no reference data were available for EPHB1, ERBB 4, JAK3, PTPRA, and PTPRM. For $4 \%$ of the tyrosine kinases and $7 \%$ of the tyrosine phosphatases, the ISH expression pattern was discordant with validation data 

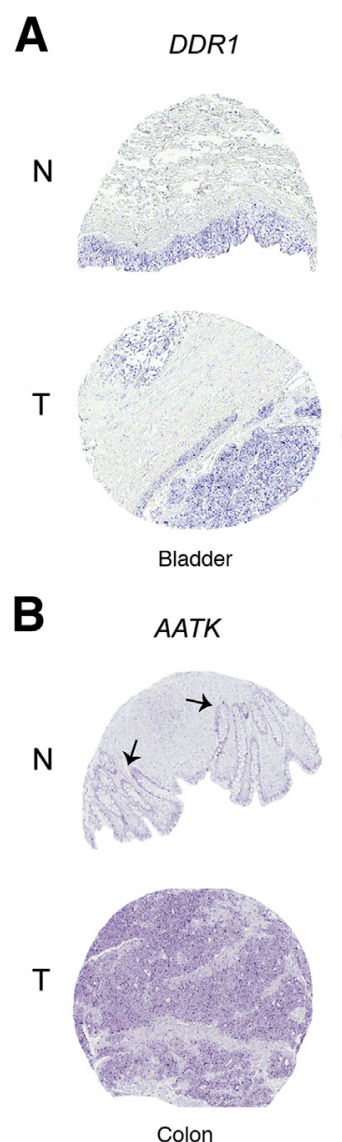

Colon

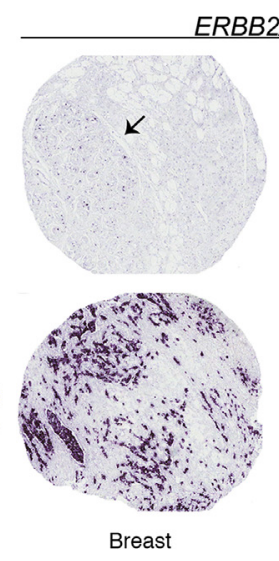

ERBB2/HER2

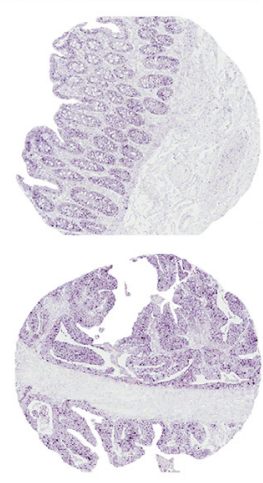

Colon
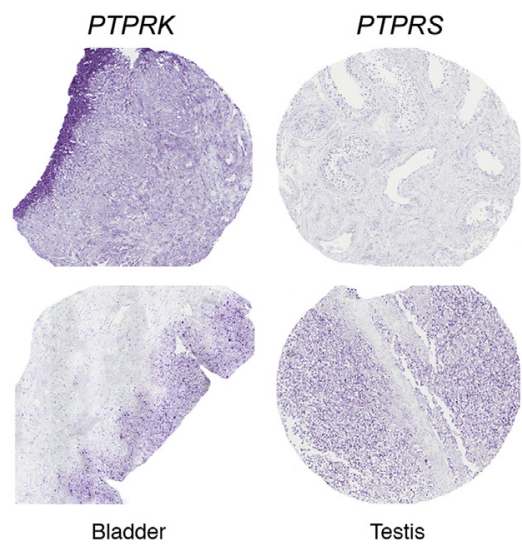

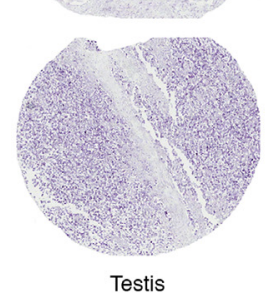

Testis

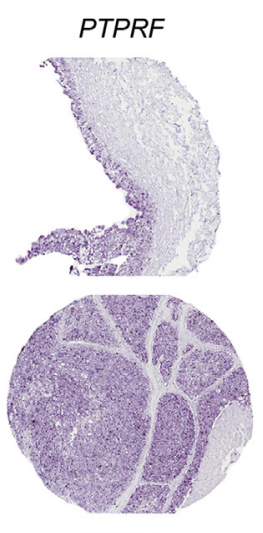

Bladder

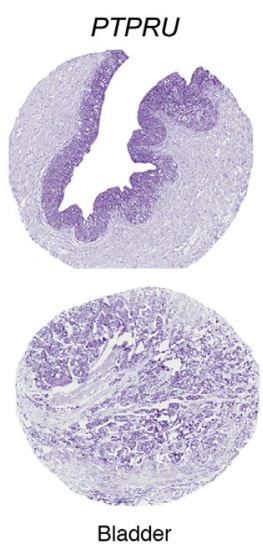

Figure 2 Expression of tyrosine kinases and phosphatases in normal and cancer tissues. A: Expression of known cancer genes. ERBB2/HER2 is strongly expressed in a subset of breast cancers, but expression is also noted in normal breast epithelium, normal colon epithelium, and colorectal cancers. Kinases and phosphatases with strong epithelial staining across several tumor types include DDR1 and PTPRF in bladder and bladder cancer. B: Expression of genes with unknown role in cancer. AATK is strongly expressed in a subset of cancers, exemplified here by colorectal cancer, and intermediate expression is observed in normal colon. For PTPRK and PTPRU intermediateto-strong epithelial expression is seen in all cancer types, shown here for bladder cancers, and expression is also observed in normal bladder. PTPRS is strongly expressed in cancers of the bladder and testis, and normal bladder, whereas normal testis is negative. Arrows indicate gene expression in the epithelium. Original magnification: $\times 8$ (A and $\mathbf{B})$, except $\times 10(\mathbf{A}$, normal bladder for DDR1 and PTPRF; B, bladder cancer for PTPRK). N, normal; $\mathrm{T}$, tumor. sources (AXL, EPHA4, YES1, MTMR2, MTMR6, and PTPN11) (Figure 1 and Supplemental Tables S1-S3 and S5-S7). Positive expression patterns consistent with prior knowledge were observed for $71 \%$ of the genes. The genes CSK, DDR1, ERBB3, JAK1, PTP4A2, PTPRF, and PTPRK would be predicted to be expressed across the tumor tissues studied here, which was also the case (Supplemental Table S3). From microarray analyses, several tyrosine kinases and phosphatases were expressed predominantly in hematologic tissues (BTK, FGR, FLT3, HCK, ITK, LCK, MATK, PTK2B, ZAP70, MTM1, PTPN6, and PTPRC), whereas others were precluded from such tissues (ABL1, EPHB3, ERBB3, FGFR2, PDGFRA, PDGFRB, PTPN3, PTPN13, PTPRD, and $P T P R F$ ), which was confirmed by ISH (Supplemental Tables S1-S3 and S5-S7).

Translocations and amplifications of driver genes may be reflected in elevated gene expression levels in an affected subset of patient tumors. ${ }^{29}$ Twenty kinome expression outliers (see Materials and Methods for definition) in gene expression array data included $D D R 2, E P H B 3, E R B B 2, F G F R 1-2, F L T 3$, INSR, KIT, NTRK2, PDGFRA, PTK6, PTK7, RET, TIE1, $T N K 1$, and YES1 in breast cancers; EGFR, ERBB2, PTK6, and PTK7 in bladder cancers; EPHA2, ERBB3, PDGFRB, PTK6, $P T K 7$, and TNK1 in colorectal cancers; ERBB3, INSR, and TIEl in renal carcinomas; and FGFRI and TNK1 in prostate cancers. Eight phosphatome gene expression outliers were identified, namely DUSP23, PTP4A1, PTPN3, PTPN12, and
PTPRT in breast cancers; PTPN13 in testicular cancers; PTPN18 in prostate cancers; and PTPRK in colorectal cancers (Supplemental Table S3). Although a partial explanation for this pattern may be derived from intertumoral variations in cell type composition, note that the frequently amplified oncogenes $E R B B 2$ and $E G F R$, along with genes known to take part in oncogenic translocations (ERBB3, FGFR1, FLT3, KIT, PDGFRs, RET, and PTPN3), are represented (Mitelman Database of Chromosome Aberrations and Gene Fusions in Cancer, http://cgap.nci.nih.gov/Chromosomes/Mitelman, last accessed May 15, 2014). Of these outlier genes in MediSapiens, 10 of 20 kinases (EGFR, EPHA2, EPHB3, ERBB2, ERBB3, INSR, $P T K 6, P T K 7, R E T$, and TNK1) and seven of eight phosphatases (DUSP23, PTP4A1, PTPN3, PTPN12, PTPN13, PTPN18, and $P T P R K)$, were observed by ISH (Supplemental Table S5).

Taken together, $91 \%$ of investigated transcripts had tumor cell in situ expression patterns in agreement with array database gene expression profiles, thus supporting the feasibility, sensitivity, and specificity of large-scale ISH to map gene expression in human FFPE tissues (Materials and Methods) (Figure 1 and Supplemental Tables S5 and S6).

\section{Compartment-Specific Gene Expression Analysis in Human Cancers}

Gene expression analyses of bulk tissue specimens will reflect the average gene expression in the different cells that constitute 


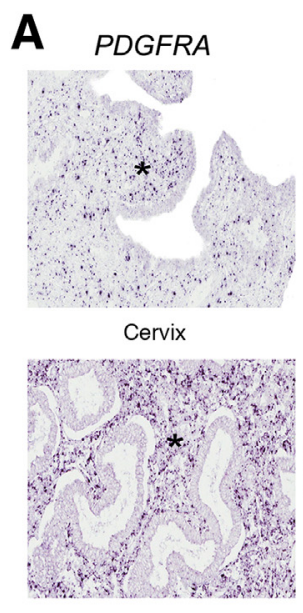

Endometrium

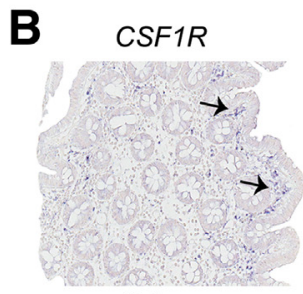

Colon

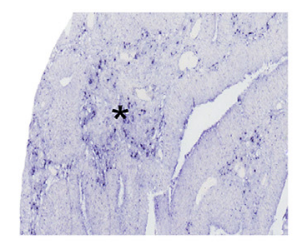

Colorectal cancer

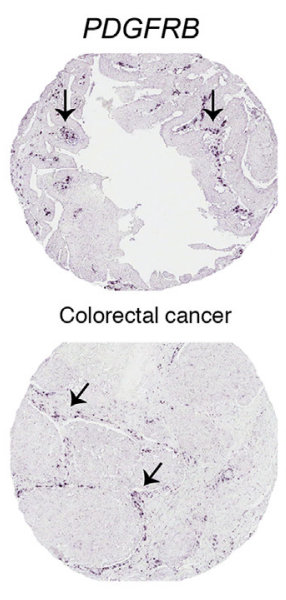

Bladder cancer

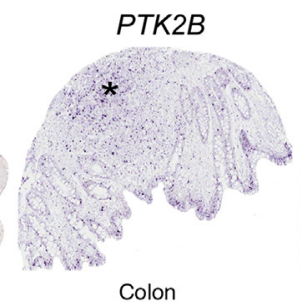

Colon

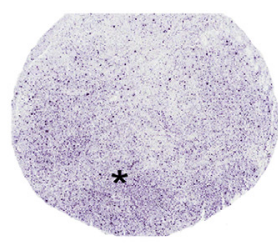

Lymph node

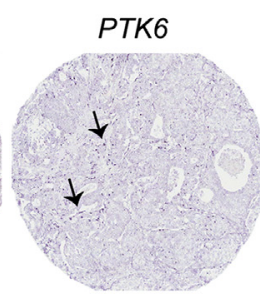

Breast cancer

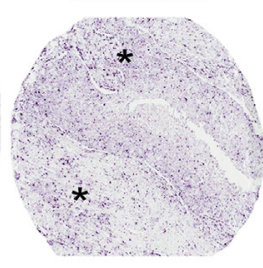

Bladder cancer
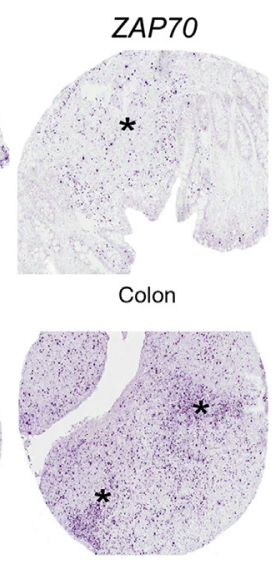

Tonsil

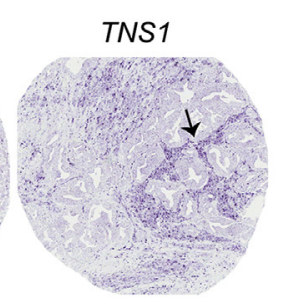

Prostate cancer

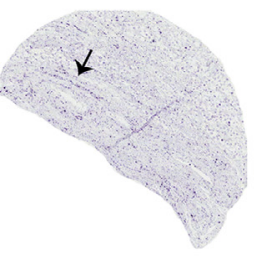

Testicular cancer

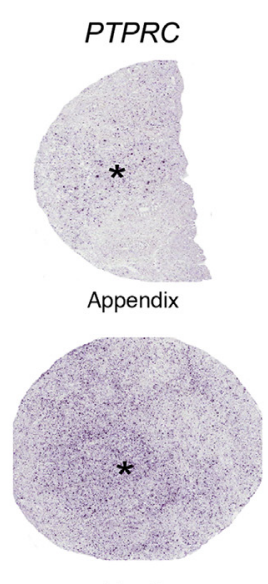

Tonsil
Figure 3 Expression of kinases and phosphatases in stromal and inflammatory cells. A: Expression in stroma. The cancer-associated fibroblast markers PDGFRA and PDGFRB are expressed in stromal compartments in cervix, endometrium and in tumors of the bladder and colorectum, respectively. Expression of PTK6 is seen in the connective tissue stroma of breast and bladder cancers. TNS1 shows stromal expression in a subset of normal tissues and in cancers, exemplified here by prostate and testicular cancers. B: Expression in inflammatory cells. Expression of CSF1R is observed in macrophages in colorectal cancers and normal colon. PTK2B is expressed in inflammatory cells of normal colon and lymph nodes. The lymphoid cell-specific kinase ZAP70 is expressed in inflammatory cells of normal colon and in many lymphatic tissues such as tonsil. Expression of PTPRC is seen in inflammatory cells of appendix and tonsil. Arrows and asterisks indicate gene expression in stromal and inflammatory cells. Original magnification: $\times 8(\mathbf{A}$, PDGFRB, PTK6, and TNS1; B, PTK2B in normal lymph nodes and $P T P R C) ; \times 10(\mathbf{B}, P T K 2 B$ in normal colon and $Z A P 70) ; \times 20($ A, PDGFRA; B, CSF1R). the sample but will not reveal gene expression in subcompartments of a tissue. To identify novel biomarkers and therapeutic targets in cancer, we sought to further locate gene expression to the tumor cells, vascular, connective tissue stroma, and inflammatory cells. We first mined SAGE libraries for transcripts enriched in breast cancer-associated myofibroblasts compared with normal myofibroblasts ${ }^{26}$ to ensure that stromal gene expression could be detected by ISH. A total of 397 SAGE tags at least fourfold enriched in cancer myofibroblasts, of which 326 corresponded to known genes, were identified. From 143 genes with $\geq 20$ SAGE tags, UniProt was used to identify 11 secreted and 7 transmembrane proteins, with evidence of ligand, receptor, or enzymatic activity, which were selected for ISH validation (Supplemental Figure S1). Of these, five genes (HTRA1, HTRA3, MXRA5, MXRA8, and SERPING1) showed tumor stroma-specific expression in cancers of the breast or colorectum (Supplemental Figure S2). Thus, the ISH approach can detect gene expression also in stromal cells.

\section{Tumor Cell Compartment}

Three-fifths of the studied genes were expressed in epithelial or tumor cell compartments (Figure 1). The spectrum of expressed tyrosine kinases and phosphatases varied between cancers of the colorectum (39\% versus 50\%), testis (35\% versus $55 \%$ ), bladder ( $38 \%$ versus $50 \%)$, breast ( $31 \%$ versus $31 \%)$, prostate (18\% versus $26 \%$ ), and kidney (15\% versus $19 \%)$. A selection of genes expressed in tumor cells is shown in Figure 2. The collagen-binding receptor DDR1 was shown to be involved in various cancers, such as those of the brain, ${ }^{30}$ and mRNA expression was seen in all epithelial tumors studied here (Figure 2A and Supplemental Table S5). Expression of the proto-oncogene $E R B B 2$ was observed in cancers of the breast, colorectum, and bladder and in normal bladder, breast, and colon (Figure 2A and Supplemental Table S5), which is relevant in the context of the observation of ERBB2 amplifications in $5 \%$ of colorectal cancers. ${ }^{20}$ PTPRF, found to be up-regulated in breast cancer, ${ }^{31}$ showed strong mRNA expression in normal epithelia and cancers of the bladder, breast, colorectum, prostate, and testis (Figure 2A and Supplemental Table S5). Several kinases and phosphatases were identified that were not previously associated with cancer. The kinase AATK, which is expressed in the developing mouse brain, ${ }^{32}$ was strongly expressed in a subset of colorectal, kidney, bladder, and testicular cancers, and intermediate expression was observed in breast and prostate cancers and normal colon (Figure 2B and 
A

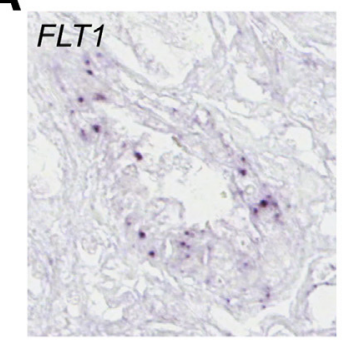

Testicular cancer

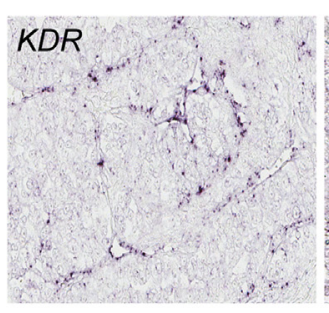

Kidney cancer

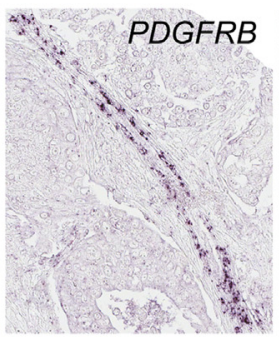

Bladder cancer

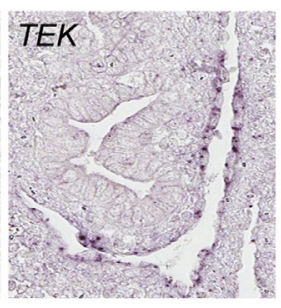

Endometrium

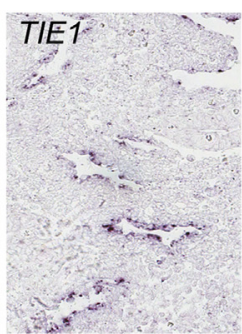

Endometrium

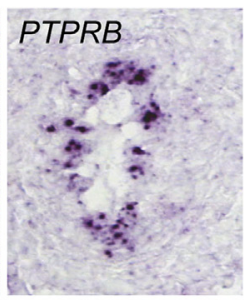

Testicular cancer

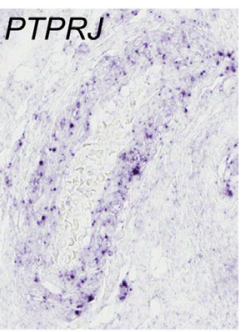

Normal testis

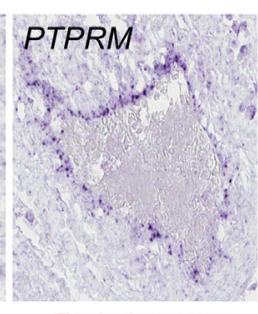

Testicular cancer
B

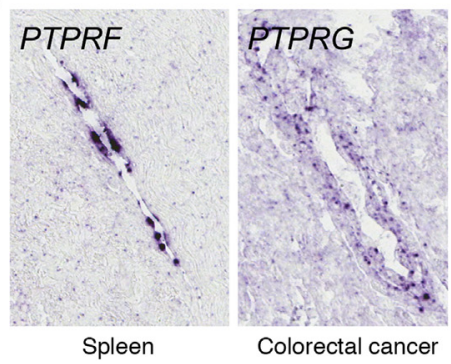

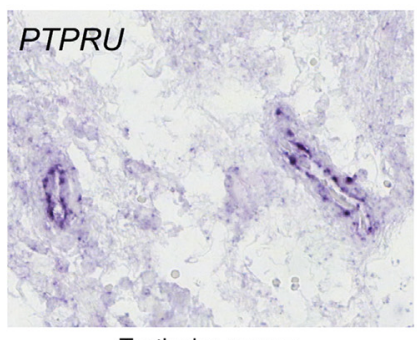

Testicular cancer

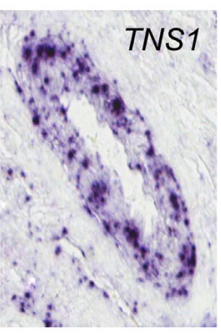

Testicular cancer
Figure 4 Expression of tyrosine kinases and phosphatases in normal and tumor vessels. A: Vascular expression of the known vessel markers FLT1, PTPRB, and PTPRM in testicular cancers; KDR in kidney cancer; TEK and TIE1 in endometrium; PDGFRB in bladder cancer; and PTPRJ in normal testis. B: Vascular expression of genes with previously unknown role in angiogenesis. PTPRF shows vascular expression in spleen, PTPRG in colorectal cancer, and PTPRU and TNS1 demonstrated vascular expression in testicular cancers. Original magnification: $\times 20($ A, PDGFRB $) ; \times 40($ A, remaining images, and $\mathbf{B})$.
Supplemental Table S5). For the candidate tumor suppressor genes PTPRK and PTPRU, ${ }^{33}$ epithelial expression in normal bladder and all studied cancers was observed (Figure $2 \mathrm{~B}$ and Supplemental Table S5). Expression of the transmembrane tyrosine phosphatase PTPRS, a potential tumor suppressor in head and neck cancers, ${ }^{34}$ was found in bladder and testicular cancers (Figure 2B and Supplemental Table S5). As expected, the tumor cell ISH expression pattern was largely in accordance with the whole tissue expression predicted by normalized gene expression array data (Supplemental Tables S3 and S5).

\section{Stromal and Inflammatory Cells}

The cancer-associated fibroblast markers PDGFRA and PDGFRB $^{15}$ were expressed in stromal compartments in tumors of the bladder and colorectum, and PDGFRB was also expressed in breast, kidney, and testicular cancers (Figure 3A and Supplemental Table S6). Strong expression was also observed in connective tissue of endometrium (Figure 3A). PTK6 is involved in regulating epithelial cell differentiation, and expression was observed in gut and skin and in cancers of the breast, colorectal, and prostate. ${ }^{35}$ Expression was also seen here in the connective tissue stroma of bladder and breast cancers (Figure $3 \mathrm{~A}$ and Supplemental Table S6). TNS1 showed stromal expression in normal testis and endometrium, and in cancers of the bladder, prostate, and testis (Figure 3A and Supplemental Table S6). Expression of the colony-stimulating factor 1 receptor (CSF1R), an important regulator of macrophage homeostasis, ${ }^{36}$ was observed in macrophages in breast and colorectal cancers and in normal breast and colon (Figure 3B and Supplemental Table S6). PTK2B, with a role in macrophage cell migration, ${ }^{37}$ was expressed in inflammatory cells of the normal colon, lymph nodes, tonsil, appendix, and spleen (Figure 3B and Supplemental Table S6). Inflammatory cells of testicular cancer, normal colon, and tissues such as lymph nodes, spleen, tonsil, appendix expressed the lymphoid cell-specific kinase ZAP70 (Figure 3B and Supplemental Table S6). PTPRC, expressed in hematopoietic cells, ${ }^{38}$ demonstrated expression in inflammatory cells of appendix, lymph nodes, and tonsil (Figure 3B and Supplemental Table S6).

\section{Blood Vessels}

One-third of the tyrosine kinases and tyrosine phosphatases, were previously found (see Reference Data Sets) to be expressed in endothelial cells or vessel walls during development or in the adult (Supplemental Tables S1 and S2). We observed vascular expression of the known vessel markers EPHA4, EPHB4, FLT1, FLT4, KDR, MET, PDGFRB, PTK2, TEK, TIE1, PTPRB, PTPRJ, and 

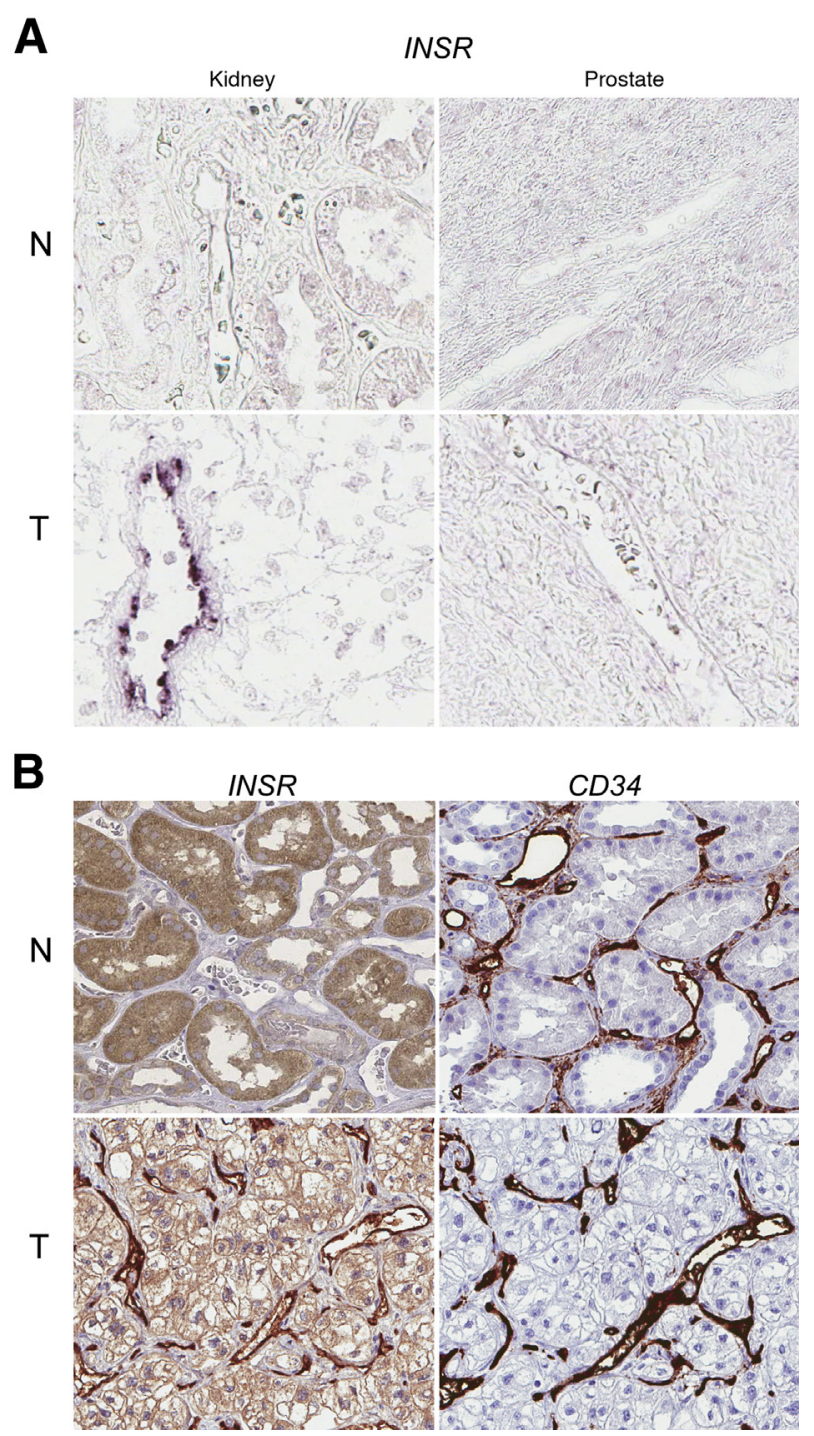

Figure 5 INSR transcript and protein is expressed in tumor vessels but not in normal vessels. A: INSR mRNA expression in vessels is observed in all evaluated tumor types but prostate cancer, with strong expression seen in kidney cancers. B: The protein expression shows similar tumor-specific staining patterns, with strong expression in kidney cancers. CD34 expression in vessels is used as a positive control. $\mathrm{N}$, normal; $\mathrm{T}$, tumor. Original magnification: $\times 20(B) ; \times 40($ A).

PTPRM in at least one normal or tumor specimen (Supplemental Table S6). Weak staining in vessels of colorectal, kidney, and testicular cancers was observed for FLT1, which is believed to be important for development of embryonic vasculature ${ }^{39}$ (Figure 4A and Supplemental Table S6). The kinase insert domain receptor (KDR), a receptor for vascular endothelial growth factor, ${ }^{40} \mathrm{had}$ vascular expression in bronchus, endometrium, pancreas, and small intestine and in cancers of the kidney and colorectum (Figure 4A and Supplemental Table S6). Vascular PDGFRB expression was observed in placenta and in cancers of the kidney, testis, and bladder (Figure 4A and Supplemental Table S6). The receptors TEK and TIE1, which are expressed in developing vascular endothelial cells, were found in endometrium where angiogenesis occurs regularly during the reproductive life of females ${ }^{41}$ (Figure 4A and Supplemental Table S6). PTPRB, previously found to be involved in maintaining and remodeling blood vessels, ${ }^{12}$ was expressed in vessels in cancers of the kidney and testis (Figure 4A and Supplemental Table S6). For PTPRJ, vascular expression was demonstrated in several normal tissues such as testis but also in testicular cancer (Figure 4A and Supplemental Table S6). Staining in vessels of colorectal, kidney, and testicular cancers was observed for PTPRM (Figure 4A and Supplemental Table S6).

Further, we observed vascular expression of several genes not previously linked to angiogenesis. These included INSR, PTPRF, PTPRG, PTPRU, and TNS1 (Figures 4B and 5A and Supplemental Table S6). The insulin receptor (INSR) was implicated in hypoxia-induced angiogenesis in the retina, but few studies have explored its role in disease. ${ }^{42,43}$ We observed strong mRNA expression of INSR in tumors, predominantly of the kidney and bladder but also in breast, colorectum, and testis but not in prostate cancer (Figure 5A and Supplemental Table S6). No vascular staining was observed in any of the evaluated normal tissues (Supplemental Table S6). The expression pattern of INSR was validated with an independent RNA probe (Supplemental Table S4). The protein expression of INSR showed similar staining patterns for the epithelial cancers studied here, with strongest expression in kidney cancer (Figure 5B). PTPRF, a potential biomarker for prostate cancer, ${ }^{44}$ was expressed in vessels of spleen and lymph nodes (Figure 4B and Supplemental Table S6). For PTPRG, a candidate tumor suppressor gene in ovarian, lung, and breast cancers, ${ }^{45}$ vascular expression was observed in cancers of the bladder, colorectum, kidney, and testis and in the placenta (Figure 4B and Supplemental Table S6). Expression in vessels of the cerebellum, normal bladder, and testicular cancer was shown for PTPRU (Figure 4B and Supplemental Table S6). Tensin-1 (TNS1), which is suggested to play a role in mesangial extracellular matrix production, ${ }^{46}$ was expressed in vessels in a subset of the cancer types analyzed and in a wide panel of normal tissues, including bladder, colon, kidney, prostate, and testis (Figure 4B and Supplemental Table S6).

Finally, several kinases and phosphatases were predominantly expressed in the central nervous system (Supplemental Table S7). Examples of such expression patterns are available in Supplemental Figure S3. Expression of NTRK3, PTPRN, PTPRS, and PTPRU was seen in Purkinje cells of the cerebellum but also in the granular layer for NTRK3 and PTK2B. Furthermore, NTRK3, PTK2B, PTPRN, PTPRS, and PTPRU were expressed in neuronal cells in the cerebral cortex, hippocampus, and lateral ventricle.

\section{Discussion}

Gene expression in cells that constitute a minor fraction of a tissue may not be detectable in analyses of bulk tissue transcriptomes. Systematic in situ gene expression analyses 
could therefore reveal important but previously overlooked gene expression in subcompartments of the tumor. This work represents the first comprehensive in situ gene expression analysis of the tyrosine kinases and phosphatases in normal tissues and epithelial cancers of the breast, prostate, colorectum, kidney, bladder, and testis. A prime advantage of this approach is high specificity, demonstrated by correspondence to previously known gene expression patterns (see Comparisons between MediSapiens and ISH) for $91 \%$ of analyzed genes, whereas the main limitation is decreased sensitivity because of the effects of fixation and embedding on tissue samples. The approach enables screening of large sets of genes to identify targets with expression patterns of interest.

Compartment-specific expression analysis can aid development of diagnostic tests and repurpose tyrosine kinase inhibitor therapies. The specific expression patterns of well-studied compartment-restricted genes such as ERBB2, PDGFRs, $C S F 1 R$, and $K D R$ indicated that such analyses were possible. Of the 90 genes that had clear expression signals and patterns concordant with validation data sources, $83 \%$ displayed expression in epithelial or tumor cells, $18 \%$ in stroma, $40 \%$ in inflammatory cells, and $20 \%$ in cells of vascular structures of normal or tumor tissues. When focusing on tumor tissues, $96 \%$ of the genes were expressed in tumor cells, $78 \%$ in vascular cells, $44 \%$ in stromal cells, and $17 \%$ in inflammatory cells. The expression of kinases and phosphatases in tumor cells will contribute the dominant component in expression analyses of bulk tumor tissues, which was confirmed by comparison of in situ expression data to gene expression array data. Elevated gene expression caused by gene amplification, such as that of $E R B B 2$ in breast cancers, is also reflected in strong ISH signals. Of the genes expressed in tumor cells, the tyrosine kinase $A A T K$ represents a little-studied potential target expressed in many forms of cancer that merits further investigation.

Furthermore, several of the novel cancer-stromal genes discovered with in silico design and SAGE libraries could represent potential targets in cancer. The human HTRA family of serine proteases consists of four members, HTRA1 to HTRA4, where HTRA1 was shown to be downregulated in several cancer forms and suggested to be a tumor suppressor that promotes cell death. ${ }^{47}$ Both HTRA1 and HTRA3 were shown to inhibit transforming growth factor- $\beta$ signaling by binding to several of its members. In addition, a statistically significant negative correlation between the levels of transforming growth factor- $\beta 1$ and HTRA1 and HTRA3, was observed in endometrial cancer, making it possible for both to be involved in inhibition of transforming growth factor- $\beta$ signaling in endometrial cancer and potentially other types of cancer. ${ }^{48}$ Expression of HTRA3 was recently demonstrated in both epithelial and stromal compartments of colorectal cancer desmoplastic invasion fronts as assessed by IHC. ${ }^{49}$ The matrix remodeling-associated protein MXRA5 was proposed as a novel biomarker in colorectal cancer. ${ }^{50}$ The MXRA8 protein is poorly characterized for cancer, but it was suggested that it may play a role in the maturation and maintenance of the blood-brain barrier. ${ }^{51}$ The gene SERPING1 encoding the $\mathrm{C} 1$ inhibitor plays an essential role in regulating the complement and contact systems and could therefore be important in the physiologic suppression of inflammation. ${ }^{52}$ The up-regulation of SERPING1 seen in the colorectal cancer stroma might be a mechanism to adjust the level of local inflammation and thereby potentiate cancer progression.

By scalable ISH, we confirmed the known stromal cell expression of PDGFRA and PDGFRB and discovered stromal expression of PTK6 and TNS1. Stromal inflammatory cell expression was limited to the known genes CSF1R, PTK2B, ZAP70, and PTPRC. However, several previously unknown vascularly expressed genes were identified, namely $P T P R F$, PTPRG, PTPRU, TNSI, and INSR. These genes provide novel potential targets for antiangiogenic approaches. Of these, INSR mRNA expression was observed in tumor endothelium but not in endothelium of normal tissues. Interestingly, INSR expression was most clearly associated with tumor angiogenesis of all genes assessed in this study, showing vascular expression in 26 tissue specimens of 112 in cancers of the kidney, bladder, breast, colorectum, and testis, including those previously known to be involved in angiogenesis (see Reference Data Sets). The underlying mechanism remains to be clarified but may reflect a strong demand for glucose in the tumor environment.

The gene families studied here are subject to major drug discovery efforts, and knowing their precise tissue expression in the adult human can guide novel therapeutic applications and can explain physiologic side effects. This study provides a rationale for investigating the insulin receptor as a target for antivascular therapies or as a vascular address for tumor homing. Pervanadate-based specific protein tyrosine phosphatase inhibitors can protect neurons after spinal cord injury ${ }^{53}$ which motivates further investigations of the neuronal expression of PTPRN, PTPRS, and PTPRU.

By defining the gene expression patterns of 127 genes of known or potential relevance to cancer, this study provides the fundamentals for comprehensive, transcriptome-wide analyses of gene expression in healthy and diseased human tissues. We have demonstrated that transcriptomic data from bulk organs and tumors, along with in situ gene expression studies in the mouse, can be used to assess ISH specificity and sensitivity, which is a major advantage to protein level analyses. Moreover, this type of large-scale analysis of gene expression can identify gene candidates with interesting expression patterns and potential clinical utility for further studies. Such efforts will be increasingly valuable with the rapid rate of discovery of novel cancer genes.

\section{Acknowledgments}

We thank IngMarie Olsson for tissue sectioning, Ann-Sofi Strand and Cane Yaka for slide scanning, and Arne Östman, 
Carl-Henrik Heldin, and Lena Claesson-Welsh for critical feedback.

\section{Supplemental Data}

Supplemental material for this article can be found at http://dx.doi.org/10.1016/j.ajpath.2015.02.019.

\section{References}

1. Greenman C, Stephens P, Smith R, Dalgliesh GL, Hunter C, Bignell G, et al: Patterns of somatic mutation in human cancer genomes. Nature 2007, 446:153-158

2. Wang Z, Shen D, Parsons DW, Bardelli A, Sager J, Szabo S, Ptak J, Silliman N, Peters BA, van der Heijden MS, Parmigiani G, Yan H, Wang TL, Riggins G, Powell SM, Willson JK, Markowitz S, Kinzler KW, Vogelstein B, Velculescu VE: Mutational analysis of the tyrosine phosphatome in colorectal cancers. Science 2004, 304: $1164-1166$

3. Bardelli A, Parsons DW, Silliman N, Ptak J, Szabo S, Saha S, Markowitz S, Willson JK, Parmigiani G, Kinzler KW, Vogelstein B, Velculescu VE: Mutational analysis of the tyrosine kinome in colorectal cancers. Science 2003, 300:949

4. Manning G, Whyte DB, Martinez R, Hunter T, Sudarsanam S: The protein kinase complement of the human genome. Science 2002, 298: $1912-1934$

5. Alonso A, Sasin J, Bottini N, Friedberg I, Osterman A, Godzik A, Hunter T, Dixon J, Mustelin T: Protein tyrosine phosphatases in the human genome. Cell 2004, 117:699-711

6. Eguchi M, Eguchi-Ishimae M, Tojo A, Morishita K, Suzuki K, Sato Y, Kudoh S, Tanaka K, Setoyama M, Nagamura F, Asano S, Kamada N: Fusion of ETV6 to neurotrophin-3 receptor TRKC in acute myeloid leukemia with $\mathrm{t}(12 ; 15)(\mathrm{p} 13 ; \mathrm{q} 25)$. Blood 1999, 93: $1355-1363$

7. Chen J, Deangelo DJ, Kutok JL, Williams IR, Lee BH, Wadleigh M, Duclos N, Cohen S, Adelsperger J, Okabe R, Coburn A, Galinsky I, Huntly B, Cohen PS, Meyer T, Fabbro D, Roesel J, Banerji L, Griffin JD, Xiao S, Fletcher JA, Stone RM, Gilliland DG: PKC412 inhibits the zinc finger 198-fibroblast growth factor receptor 1 fusion tyrosine kinase and is active in treatment of stem cell myeloproliferative disorder. Proc Natl Acad Sci U S A 2004, 101:14479-14484

8. Demetri GD, von Mehren M, Blanke CD, Van den Abbeele AD, Eisenberg B, Roberts PJ, Heinrich MC, Tuveson DA, Singer S, Janicek M, Fletcher JA, Silverman SG, Silberman SL, Capdeville R, Kiese B, Peng B, Dimitrijevic S, Druker BJ, Corless C, Fletcher CD, Joensuu H: Efficacy and safety of imatinib mesylate in advanced gastrointestinal stromal tumors. N Engl J Med 2002, 347:472-480

9. O'Brien SG, Guilhot F, Larson RA, Gathmann I, Baccarani M, Cervantes F, Cornelissen JJ, Fischer T, Hochhaus A, Hughes T, Lechner K, Nielsen JL, Rousselot P, Reiffers J, Saglio G, Shepherd J, Simonsson B, Gratwohl A, Goldman JM, Kantarjian H, Taylor K, Verhoef G, Bolton AE, Capdeville R, Druker BJ; IRIS Investigators: Imatinib compared with interferon and low-dose cytarabine for newly diagnosed chronic-phase chronic myeloid leukemia. N Engl J Med 2003, 348:994-1004

10. Slamon DJ, Leyland-Jones B, Shak S, Fuchs H, Paton V, Bajamonde A, Fleming T, Eiermann W, Wolter J, Pegram M, Baselga J, Norton L: Use of chemotherapy plus a monoclonal antibody against HER2 for metastatic breast cancer that overexpresses HER2. N Engl J Med 2001, 344: 783-792

11. Ostman A, Heldin CH: PDGF receptors as targets in tumor treatment. Adv Cancer Res 2007, 97:247-274
12. Baumer S, Keller L, Holtmann A, Funke R, August B, Gamp A, Wolburg H, Wolburg-Buchholz K, Deutsch U, Vestweber D: Vascular endothelial cell-specific phosphotyrosine phosphatase (VE-PTP) activity is required for blood vessel development. Blood 2006, 107:4754-4762

13. Holopainen T, Lopez-Alpuche V, Zheng W, Heljasvaara R, Jones D, He Y, Tvorogov D, D'Amico G, Wiener Z, Andersson LC, Pihlajaniemi T, Min W, Alitalo K: Deletion of the endothelial Bmx tyrosine kinase decreases tumor angiogenesis and growth. Cancer Res 2012, 72:3512-3521

14. Augustin HG, Koh GY, Thurston G, Alitalo K: Control of vascular morphogenesis and homeostasis through the angiopoietin-Tie system. Nat Rev Mol Cell Biol 2009, 10:165-177

15. Ostman A: PDGF receptors-mediators of autocrine tumor growth and regulators of tumor vasculature and stroma. Cytokine Growth Factor Rev 2004, 15:275-286

16. Ferrara N, Gerber HP, LeCouter J: The biology of VEGF and its receptors. Nat Med 2003, 9:669-676

17. Saylor PJ, Escudier B, Michaelson MD: Importance of fibroblast growth factor receptor in neovascularization and tumor escape from antiangiogenic therapy. Clin Genitourin Cancer 2012, 10:77-83

18. Sjöblom T, Jones S, Wood LD, Parsons DW, Lin J, Barber TD, Mandelker D, Leary RJ, Ptak J, Silliman N, Szabo S, Buckhaults P, Farrell C, Meeh P, Markowitz SD, Willis J, Dawson D, Willson JK, Gazdar AF, Hartigan J, Wu L, Liu C, Parmigiani G, Park BH, Bachman KE, Papadopoulos N, Vogelstein B, Kinzler KW, Velculescu VE: The consensus coding sequences of human breast and colorectal cancers. Science 2006, 314:268-274

19. Wood LD, Parsons DW, Jones S, Lin J, Sjoblom T, Leary RJ, et al: The genomic landscapes of human breast and colorectal cancers. Science 2007, 318:1108-1113

20. Comprehensive molecular characterization of human colon and rectal cancer. Nature 2012, 487:330-337

21. Kilpinen S, Autio R, Ojala K, Iljin K, Bucher E, Sara H, Pisto T, Saarela M, Skotheim RI, Bjorkman M, Mpindi JP, Haapa-Paananen S, Vainio P, Edgren H, Wolf M, Astola J, Nees M, Hautaniemi S, Kallioniemi O: Systematic bioinformatic analysis of expression levels of 17,330 human genes across 9,783 samples from 175 types of healthy and pathological tissues. Genome Biol 2008, 9:R139

22. Hawrylycz MJ, Lein ES, Guillozet-Bongaarts AL, Shen EH, Ng L, Miller JA, et al: An anatomically comprehensive atlas of the adult human brain transcriptome. Nature 2012, 489:391-399

23. Lein ES, Hawrylycz MJ, Ao N, Ayres M, Bensinger A, Bernard A, et al: Genome-wide atlas of gene expression in the adult mouse brain. Nature 2007, 445:168-176

24. Kiflemariam S, Andersson S, Asplund A, Ponten F, Sjoblom T: Scalable in situ hybridization on tissue arrays for validation of novel cancer and tissue-specific biomarkers. PLoS One 2012, 7:e32927

25. Paavilainen L, Edvinsson A, Asplund A, Hober S, Kampf C, Ponten F, Wester K: The impact of tissue fixatives on morphology and antibody-based protein profiling in tissues and cells. J Histochem Cytochem 2010, 58:237-246

26. Allinen M, Beroukhim R, Cai L, Brennan C, Lahti-Domenici J, Huang H, Porter D, Hu M, Chin L, Richardson A, Schnitt S, Sellers WR, Polyak K: Molecular characterization of the tumor microenvironment in breast cancer. Cancer Cell 2004, 6:17-32

27. Krizman DB, Wagner L, Lash A, Strausberg RL, Emmert-Buck MR: The Cancer Genome Anatomy Project: EST sequencing and the genetics of cancer progression. Neoplasia 1999, 1:101-106

28. Kampf C, Olsson I, Ryberg U, Sjostedt E, Ponten F: Production of tissue microarrays, immunohistochemistry staining and digitalization within the human protein atlas. J Vis Exp 2012, 31:pii:3620

29. Tomlins SA, Rhodes DR, Perner S, Dhanasekaran SM, Mehra R, Sun XW, Varambally S, Cao X, Tchinda J, Kuefer R, Lee C, Montie JE, Shah RB, Pienta KJ, Rubin MA, Chinnaiyan AM: Recurrent fusion of TMPRSS2 and ETS transcription factor genes in prostate cancer. Science 2005, 310:644-648 
30. Weiner HL, Huang H, Zagzag D, Boyce H, Lichtenbaum R, Ziff EB: Consistent and selective expression of the discoidin domain receptor-1 tyrosine kinase in human brain tumors. Neurosurgery 2000, 47: $1400-1409$

31. Levea CM, McGary CT, Symons JR, Mooney RA: PTP LAR expression compared to prognostic indices in metastatic and nonmetastatic breast cancer. Breast Cancer Res Treat 2000, 64:221-228

32. Tomomura M, Hasegawa Y, Hashikawa T, Tomomura A, Yuzaki M, Furuichi T, Yano R: Differential expression and function of apoptosis-associated tyrosine kinase (AATYK) in the developing mouse brain. Brain Res Mol Brain Res 2003, 112:103-112

33. Scrima M, De Marco C, De Vita F, Fabiani F, Franco R, Pirozzi G, Rocco G, Malanga D, Viglietto G: The nonreceptor-type tyrosine phosphatase PTPN13 is a tumor suppressor gene in non-small cell lung cancer. Am J Pathol 2012, 180:1202-1214

34. Morris LG, Taylor BS, Bivona TG, Gong Y, Eng S, Brennan CW, Kaufman A, Kastenhuber ER, Banuchi VE, Singh B, Heguy A, Viale A, Mellinghoff IK, Huse J, Ganly I, Chan TA: Genomic dissection of the epidermal growth factor receptor (EGFR)/PI3K pathway reveals frequent deletion of the EGFR phosphatase PTPRS in head and neck cancers. Proc Natl Acad Sci U S A 2011, 108:19024-19029

35. Brauer PM, Tyner AL: Building a better understanding of the intracellular tyrosine kinase PTK6-BRK by BRK. Biochim Biophys Acta 2010, 1806:66-73

36. Stanley ER, Cifone M, Heard PM, Defendi V: Factors regulating macrophage production and growth: identity of colony-stimulating factor and macrophage growth factor. J Exp Med 1976, 143:631-647

37. Okigaki M, Davis C, Falasca M, Harroch S, Felsenfeld DP, Sheetz MP, Schlessinger J: Pyk2 regulates multiple signaling events crucial for macrophage morphology and migration. Proc Natl Acad Sci U S A 2003, 100:10740-10745

38. Hermiston $\mathrm{ML}, \mathrm{Xu} \mathrm{Z}$, Weiss A: CD45: a critical regulator of signaling thresholds in immune cells. Annu Rev Immunol 2003, 21: 107-137

39. Fong GH, Rossant J, Gertsenstein M, Breitman ML: Role of the Flt-1 receptor tyrosine kinase in regulating the assembly of vascular endothelium. Nature 1995, 376:66-70

40. Quinn TP, Peters KG, De Vries C, Ferrara N, Williams LT: Fetal liver kinase 1 is a receptor for vascular endothelial growth factor and is selectively expressed in vascular endothelium. Proc Natl Acad Sci U S A 1993, 90:7533-7537

41. Gargett CE, Rogers PA: Human endometrial angiogenesis. Reproduction 2001, 121:181-186

42. Kondo T, Vicent D, Suzuma K, Yanagisawa M, King GL, Holzenberger M, Kahn CR: Knockout of insulin and IGF-1 receptors on vascular endothelial cells protects against retinal neovascularization. J Clin Invest 2003, 111:1835-1842

43. Rensing KL, Houttuijn Bloemendaal FM, Weijers EM, Richel DJ, Buller HR, Koolwijk P, van der Loos CM, Twickler TB, von der Thusen JH: Could recombinant insulin compounds contribute to adenocarcinoma progression by stimulating local angiogenesis? Diabetologia 2010, 53:966-970

44. Whitmore TE, Peterson A, Holzman T, Eastham A, Amon L, McIntosh M, Ozinsky A, Nelson PS, Martin DB: Integrative analysis of N-linked human glycoproteomic data sets reveals PTPRF ectodomain as a novel plasma biomarker candidate for prostate cancer. J Proteome Res 2012, 11:2653-2665

45. Vezzalini M, Mombello A, Menestrina F, Mafficini A, Della Peruta M, van Niekerk C, Barbareschi M, Scarpa A, Sorio C: Expression of transmembrane protein tyrosine phosphatase gamma (PTPgamma) in normal and neoplastic human tissues. Histopathology 2007, 50:615-628

46. Yamashita M, Horikoshi S, Asanuma K, Takahara H, Shirato I, Tomino Y: Tensin is potentially involved in extracellular matrix production in mesangial cells. Histochem Cell Biol 2004, 121: $245-254$

47. Zurawa-Janicka D, Skorko-Glonek J, Lipinska B: HtrA proteins as targets in therapy of cancer and other diseases. Expert Opin Ther Targets 2010, 14:665-679

48. Narkiewicz J, Lapinska-Szumczyk S, Zurawa-Janicka D, SkorkoGlonek J, Emerich J, Lipinska B: Expression of human HtrA1, HtrA2, HtrA3 and TGF-beta1 genes in primary endometrial cancer. Oncol Rep 2009, 21:1529-1537

49. Karagiannis GS, Treacy A, Messenger D, Grin A, Kirsch R, Riddell RH, Diamandis EP: Expression patterns of bone morphogenetic protein antagonists in colorectal cancer desmoplastic invasion fronts. Mol Oncol 2014, 8:1240-1252

50. Wang GH, Yao L, Xu HW, Tang WT, Fu JH, Hu XF, Cui L, Xu XM: Identification of MXRA5 as a novel biomarker in colorectal cancer. Oncol Lett 2013, 5:544-548

51. Yonezawa T, Ohtsuka A, Yoshitaka T, Hirano S, Nomoto H, Yamamoto K, Ninomiya Y: Limitrin, a novel immunoglobulin superfamily protein localized to glia limitans formed by astrocyte endfeet. Glia 2003, 44:190-204

52. Davis AE 3rd, Lu F, Mejia P: C1 inhibitor, a multi-functional serine protease inhibitor. Thromb Haemost 2010, 104:886-893

53. Nakashima S, Arnold SA, Mahoney ET, Sithu SD, Zhang YP, D'Souza SE, Shields CB, Hagg T: Small-molecule protein tyrosine phosphatase inhibition as a neuroprotective treatment after spinal cord injury in adult rats. J Neurosci 2008, 28:7293-7303 